\title{
Determinants of distance walked during the six-minute walk test in patients undergoing cardiac surgery at hospital discharge
}

Géssica Uruga Oliveira', Vitor Oliveira Carvalho ${ }^{2,3}$, Lucas Pereira de Assis Cacau ${ }^{4}$, Amaro Afrânio de Araújo Filho ${ }^{4}$, Manoel Luiz de Cerqueira Neto ${ }^{2}$, Walderi Monteiro da Silva Junior ${ }^{2}$, Telma Cristina Fontes Cerqueira ${ }^{5}$ and Valter Joviniano de Santana Filho $2,5^{*}$

\begin{abstract}
Introduction: The aim of this study was to identify the determinants of distance walked in six-minute walk test (6MWD) in patients undergoing cardiac surgery at hospital discharge.

Methods: The assessment was performed preoperatively and at discharge. Data from patient records were collected and measurement of the Functional Independence Measure (FIM) and the Nottingham Health Profile $(\mathrm{NHP})$ were performed. The six-minute walk test (6MWT) was performed at discharge. Patients undergoing elective cardiac surgery, coronary artery bypass grafting or valve replacement were eligible. Patients older than 75 years who presented arrhythmia during the protocol, with psychiatric disorders, muscular or neurological disorders were excluded from the study.

Results: Sixty patients (44.26\% male, mean age $51.53 \pm 13$ years) were assessed. In multivariate analysis the following variables were selected: type of surgery $(P=0.001)$, duration of cardiopulmonary bypass (CPB) $(P=0.001)$, Functional Independence Measure - FIM (0.004) and body mass index - BMI (0.007) with $r=0.91$ and $r 2=0.83$ with $P<0.001$. The equation derived from multivariate analysis: $6 \mathrm{MWD}=$ Surgery (89.42) + CPB (1.60) + MIF (2.79) - BMI (7.53) - 127.90.
\end{abstract}

Conclusion: In this study, the determinants of 6MWD in patients undergoing cardiac surgery were: the type of surgery, CPB time, functional capacity and body mass index.

Keywords: Six-minute walk test, Exercise, Cardiac surgery, Physiotherapy

\section{Background}

Cardiac surgery is a well-established procedure worldwide due to its safety and effectiveness in treating cardiac patients. Among cardiac surgery, we highlight the CABG and valvar replacement [1].

Despite the undeniable efficiency, recovery of functional capacity of patients in the postoperative period of cardiac surgery is one of the important aspects and discussed in literature [2]. A simple and efficient method to directly assess the functional capacity of the patient in the postoperative period of cardiac surgery is through

\footnotetext{
* Correspondence: vjsf@infonet.com.br

${ }^{2}$ Departamento de Fisioterapia da Universidade Federal de Sergipe - UFS,

Aracaju, SE, Brazil

${ }^{5}$ Departamento de Fisioterapia da Universidade Federal de Sergipe - UFS,

Lagarto, SE, Brazil

Full list of author information is available at the end of the article
}

the six-minute walk test (6MWT). To perform the test, patients are instructed to walk as far as possible in six minutes and this maxim distance walked reflects his physical ability $[3,4]$. It is known that the distance $\geq 300 \mathrm{~m}$ covered in the 6MWT is a predictor of increased survival at 5 years of follow-up of elderly patients undergoing cardiac surgery [5]. Recently, the ability or inability to perform the 6MWT in patients undergoing cardiac surgery in pre-hospital discharge was used as a predictor of risk of postoperative complications and mortality [6,7]. Nevertheless, this is still unclear in the literature the determinants factors of the distance walked in the six-minute walk test (6MWD).

Given that the use of the 6MWT is growing in population undergoing cardiac surgery, the aim of this study was to identify the determinants of 6MWD at hospital 
discharge in patients undergoing cardiac surgery. In addition to establishing a reference equation and test its reliability.

\section{Methods}

\section{Study design}

This is an observational study that aimed to investigate the determinants of 6MWD in patients undergoing cardiac surgery.

This study was performed at a tertiary cardiology hospital in Brazil. The patients' assessments were performed in two stages: preoperatively and at discharge. Data from patients' records were collected, spirometry and implementation of the Functional Independence Measure (FIM) questionnaire and the Nottingham Health Profile were performed. The 6MWT was performed only at the time of patient's discharge.

\section{Studied population}

Patients undergoing elective cardiac surgery, coronary artery bypass grafting and/or valve replacement were eligible for this study. Patients aged over 75 years; who required more than 24 hours of mechanical ventilation or surgical reintervention, patients who had arrhythmia during mechanical ventilation protocol, patients with psychiatric disorders, neurological or muscular disorders that prevented the completion of the protocol were excluded from the study. Patients were recruited from a tertiary hospital cardiology from December 2011 to August 2013. The patients' characteristics are listed in Table 1.

This study was approved by the Research Ethics Committee of the Federal University of Sergipe under protocol number CAAE-0180.0.107.107-11. All patients signed written informed consent before participation.

\section{Variables considered potentially associated with the 6MWD}

Age, gender, type of surgery (coronary artery bypass grafting (CABG) or valve replacement (non-CABG)), days of hospitalization in the intensive care unit (ICU), length of hospitalization, duration of mechanical ventilation (MV), cardiopulmonary bypass (CPB) time, quality of life (Nottingham Health Profile, functional capacity (FIM), body mass index (BMI), preoperative left ventricular ejection fraction (LVEF), forced vital capacity (FVC), forced expiratory volume in one second (FEV1), presence of diabetes and hemoglobin dosage.

\section{The six-minute walk test (6MWT)}

The 6MWT was performed according to American Thoracic Society [8]. A plan corridor with $30 \mathrm{~m}$ and free of obstacles was used. While the patient remained seated, we assessed respiratory rate, heart rate, blood
Table 1 Characteristics of study participants (60 patients)

\begin{tabular}{ll}
\hline Variable & \\
\hline Age (years) & $51 \pm 13$ \\
Gender & \\
Men/Women (\%) & $44.2 / 55.7$ \\
Surgery & \\
CABG (\%) & 44.2 \\
Replacement/Valverepair (\%) & 55.7 \\
BMI (Kg/m2) & 24.8 \\
ICU time (days) & $3.5 \pm 6$ \\
Time of hospital stay (days) & $11.3 \pm 6$ \\
Preoperative LV ejectionfraction (\%) & $60 \pm 14$ \\
CPB time (min) & $93 \pm 27$ \\
FVC (Pre) & $1.20 \pm 0.4$ \\
FVC (Discharge) & $1.63 \pm 0.5$ \\
FVE1 (Pre) & $2.02 \pm 0.6$ \\
FVE1 (Discharge) & $2.54 \pm 0.8$ \\
NHP (Pre) & $158.06 \pm 99.01$ \\
NHP (Discharge) & $160.70 \pm 100$ \\
FIM (Pre) & $123 \pm 4$ \\
FIM (Discharge) & $115 \pm 10.8$ \\
DM (\%) & 20 \\
\hline BMI body mass ndex, CPBcardopulnonay byps, & \\
\hline &
\end{tabular}

BMI: body mass index, CPB: cardiopulmonary bypass, ICU: intensive care unit, LV: left ventricle; FVC - Forced Vital Capacity; FVE1 - Forced expiratory volume in one second; DM: diabetes mellitus. Data expressed in percentage of occurrence is the prevalence in this sample.

pressure, peripheral oxygen saturation and subjective sensation of dyspnea (modified Borg scale) [8].

\section{Assessment of pulmonary function}

Pulmonary function was characterized by spirometry using a portable spirometer manufactured by Clement Clarke ONE FLOW ${ }^{\circ}$. The forced vital capacity maneuvers were performed following the technical procedures, criteria for acceptability and reproducibility, as recommended by ATS [9].

\section{Functional capacity}

Functional capacity was assessed by the Functional Independence Measure (FIM), which is part of the Uniform Data System for Medical Rehabilitation and has been widely used and accepted as a measure of functional assessment [10] and is validated in Brazil [11]. The FIM assesses the independence and assistance demanded by a person to perform a range of motor and cognitive tasks of daily living. Among the assessed activities we mention self-care, transfers, locomotion, sphincter control, communication and social interaction $[10,11]$.

The FIM has 18 items of assessment and is composed of two fields: motor and cognitive. The motor field is 
composed of 13 items and divided into four categories: personal care, sphincter control, mobility/transfer, locomotion. The cognitive field consists of 5 items, in two categories: communication and social cognition. For each item is assigned a score ranging from $1=$ full assistance, $2=$ high assistance, $3=$ moderate assistance, $4=\mathrm{min}$ imal assistance, 5 = supervision, $6=$ modified independence and $7=$ complete independence. Thus the total score ranges from 18 (total dependence) to 126 (complete independence) $[11,12]$.

\section{Assessment of quality of life}

To assess the quality of life we used the Nottingham Health Profile, a generic instrument originally developed to assess quality of life in patients with chronic diseases [13]. This is a self-administered questionnaire consisting of 38 items, based on the classification of disability described by the World Health Organization. Using a language easy to interpret, the NHP provides a simple measure of physical, social and emotional health of the individual being considered clinically valid for distinguishing patients with different levels of impairment and to detect significant changes in the framework of the patient over time [14].

\section{Data collected from the patient's records}

Type of surgery, duration of mechanical ventilation, $\mathrm{CPB}$ time, days of hospitalization, days of ICU admission, LVEF, hemoglobin and comorbidities (high blood pressure (hypertension)), diabetes and dyslipidemia).

\section{Statistical analysis}

Data are presented as mean and standard deviation. We used univariate analysis to select the variables to be used in the multivariate analysis model. For univariate analysis, we consider a level of significance less than $20 \%(P<0.20)$. In multivariate analysis, the nonsignificant variables were excluded from the model one at a time manually following the criterion of the highest $P$ value. The model was determined when all the variables were presented with a level of significance less than 5\% $(P<0.05)$. The dependent variable was the distance in the six-minute walk test. After the analysis, we establish an equation with the selected variables.

The variables considered for the univariate analysis were: age, gender, type of surgery (CABG or nonCABG), ICU days, days of hospitalization, duration of $\mathrm{CPB}$, duration of MV, pre-LVEF, discharge NHP, discharge motor FIM, total discharge FIM, pre-FVC, discharge FVC, pre-FEV1, discharge FEV1, BMI, presence of diabetes and hemoglobin.

The reliability of our equation was assessed using the Bland and Altman representation for comparison of the measured and predicted distance with the equation proposed in a second group of 6 patients, $10 \%$ of the sample that generated the equation [15].

\section{Results}

Ninety-four patients undergoing cardiac surgery were selected, 34 were excluded due to discharge before the 6MWT, MV time greater than 24 hours, need for reoperation, death and denial (Figure 1). The characteristics of the 60 patients assessed are summarized in Table 1.

The 6MWT was well tolerated by all patients and no test was interrupted before completing 6 minutes. The mean 6MWD was $260.20 \pm 89.20$ meters.

Univariate analysis selected the following variables considered in the multivariate analysis: age $(P=0.001)$, type of surgery $(P=0.037)$, ICU days $(P=0.037), \mathrm{CPB}$ time $(P=0.13)$, total FIM at discharge $(P=0.074)$, BMI $(P=0.20)$ and presence of diabetes $(P=0.20)$.

The following variables, age $(P=0.69)$, presence of diabetes $(P=0.64)$ and ICU days $(P=0.99)$ (in order of exclusion) were excluded from the multivariate analysis model.

Multivariate analysis selected the following variables: type of surgery $(P=0.001)$, CPB time $(P=0.001)$, total FIM (0.004) and BMI (0.007), considering $\mathrm{r}=0.91$ and r2 $=0.83$ with $P<0.001$.

The equation derived from the multivariate analysis was:

$$
\begin{aligned}
6 \mathrm{MWD}= & \text { Surgery }(89.42)+\mathrm{CPB}(1.60) \\
& + \text { total FIM }(2.79)-\mathrm{BMI}(7.53)-127.90
\end{aligned}
$$

"considering 6MWT distance in meters; surgery type: 1 to $\mathrm{CABG}$ and 2 to non-CABG; $\mathrm{CPB}$ time in minutes; BMI in $\mathrm{kg} / \mathrm{m}^{2}$.

Given that the type of surgery was the variable that most influenced the distance covered on the 6MWT, we decided to compare the profile of our patients who had undergone $C A B G$ and those who had not undergone CABG. The CABG group had higher age $(P<0.0001)$ and a higher percentage of risk factors such as hypertension, diabetes, dyslipidemia, history of smoking and alcohol consumption (Table 2).

\section{Equation reliability}

The mean 6MWD assessed in a second group of patients $(n=6)$, used to test the reliability of the equation generated was $330.14 \pm 55.51$ meters, which is $106 \pm 13 \%$ of predicted value, calculated with our equation. The correlation between predicted and measured values was significant $(\mathrm{r}=0.76, P<0.05)$.

Figure 2 shows the Bland Altman [15] graphical representation of the comparison between the achieved $6 \mathrm{MWT}$ in the second group of patients and predicted $6 \mathrm{MWD}$ by the proposed equation. 


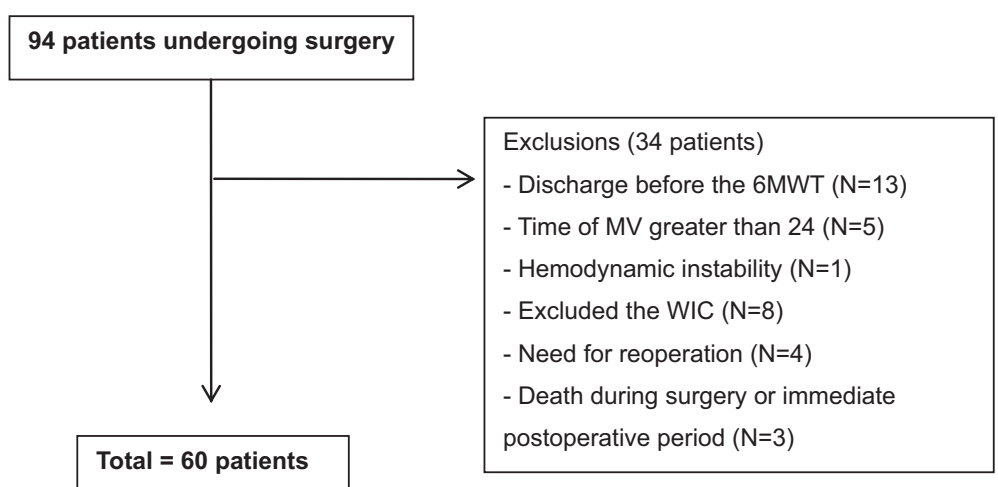

Figure 1 Flowchart of study patients.

\section{Discussion}

The main finding of this study was that the 6MWD at hospital discharge in patients undergoing cardiac surgery was determined by the type of surgery (CABG or not), CPB time, total FIM and BMI.

Our study is the first to investigate the determinants of 6MWD in patients undergoing cardiac surgery using noninvasive and easy variables to be collected in clinical practice. Moreover, we were the first to propose and test a predictive $6 \mathrm{MWD}$ equation at hospital discharge in patients undergoing cardiac surgery.

In our study, we observed that the 6MWD was directly associated with the type of surgery, $\mathrm{CPB}$ and the

Table $\mathbf{2}$ Characteristics of study participants stratified on the type of surgery

\begin{tabular}{lccc}
\hline & CABG & Non-CABG & $P$ \\
\hline Age & $57.88 \pm 8.7$ & $45.66 \pm 12.5$ & $<0.0001^{*}$ \\
Gender & & & \\
Men & $51.8 \%$ & $33.3 \%$ & \\
BMI & $24.53 \pm 6.8$ & $25.24 \pm 4.7$ & 0.99 \\
ICU time (days) & $2.3 \pm 0.4$ & $2.8 \pm 0.7$ & $0.006^{*}$ \\
Time of hospital stay (days) & $10 \pm 2.7$ & $14.7 \pm 10.97$ & 0.12 \\
Preoperative LVEF (\%) & $60.25 \pm 13.0$ & $62.54 \pm 10.63$ & 0.87 \\
CPB time (mim) & $98.33 \pm 40.99$ & $83.38 \pm 28.47$ & 0.1088 \\
Time of MV (hours) & $11.54 \pm 3.5$ & $11.04 \pm 5.22$ & 0.1945 \\
Ex -smokers (\%) & 51.8 & 21.2 & $0.0005^{*}$ \\
Drinker/Ex-drinker (\%) & 48.1 & 30.3 & 0.057 \\
Diabetics (\%) & 40.7 & 3 & $<0.0001$ \\
HBP (\%) & 74 & 48.4 & 0.026 \\
Dyslipidemia (\%) & 33.3 & 18.5 & 0.05 \\
\hline CABG: coronary artery bypass & & &
\end{tabular}

CABG: coronary artery bypass grafting, BMl: body mass index, CPB: cardiopulmonary bypass, ICU: intensive care unit, LVEF: left ventricular ejection fraction; MV: Mechanical ventilation; HBP: systemic arterial hypertension,T Test was performed for parametric variables, Mann-Whitney test for nonparametric variables and $C h i-S q u a r e d$ test for categoric variables. $\left({ }^{*} P<0.05\right)$. Data expressed in percentage of occurrence is the prevalence in this sample.
Functional Independence Measure (FIM). Furthermore, the 6MWD was negatively associated with BMI.

Our results showed the type of surgery (CABG or non-CABG) as the main predictive variable for the $6 \mathrm{MWD}$. The literature is controversial regarding the influence of the type of surgery and physical capacity in postoperative $[6,16]$. Our study, however, shows a better performance for patients undergoing non-CABG surgery. Studies show that individuals who exhibit a combination of cardiovascular risk factors have a poorer performance on the $6 \mathrm{MWT}$ compared to healthy individuals $[17,18]$. In general, in our study, it was observed that patients undergoing CABG showed a higher percentage of cardiovascular risk factors, which may indeed be negatively influencing walking ability in this group.

In our study, the influence of CPB in 6MWD was minimal, but positive, and this may have occurred because of the more severe patients were excluded from the group. There are no records in the studies assessing the influence of $\mathrm{CPB}$ on walking capacity literature, but some studies show that CPB does not influence the outcome of pulmonary function and postoperative gasometry $[19,20]$.

The negative influence of BMI is well established in the field of exercise physiology. Enrigth, et al. [21]. noted that overweight may influence walking and increase the workload. Obesity increases the workload for a given amount of exercise, resulting in a lower 6MWD in patients with higher body weight or BMI.

The influence of gender on the 6MWD has been described in some studies $[18,21,22]$, however in our study gender was not a predictive variable of the 6MWD in our population. The absence of gender as a predictor of 6MWD is consistent with observations from other studies $[18,23]$ which showed that after adjusting for height and weight, there is no difference in MWD between men and women.

Other studies showed that spirometric values showed a strong positive correlation with the 6MWD in healthy adults $[24,25]$ and in our study the pulmonary function 


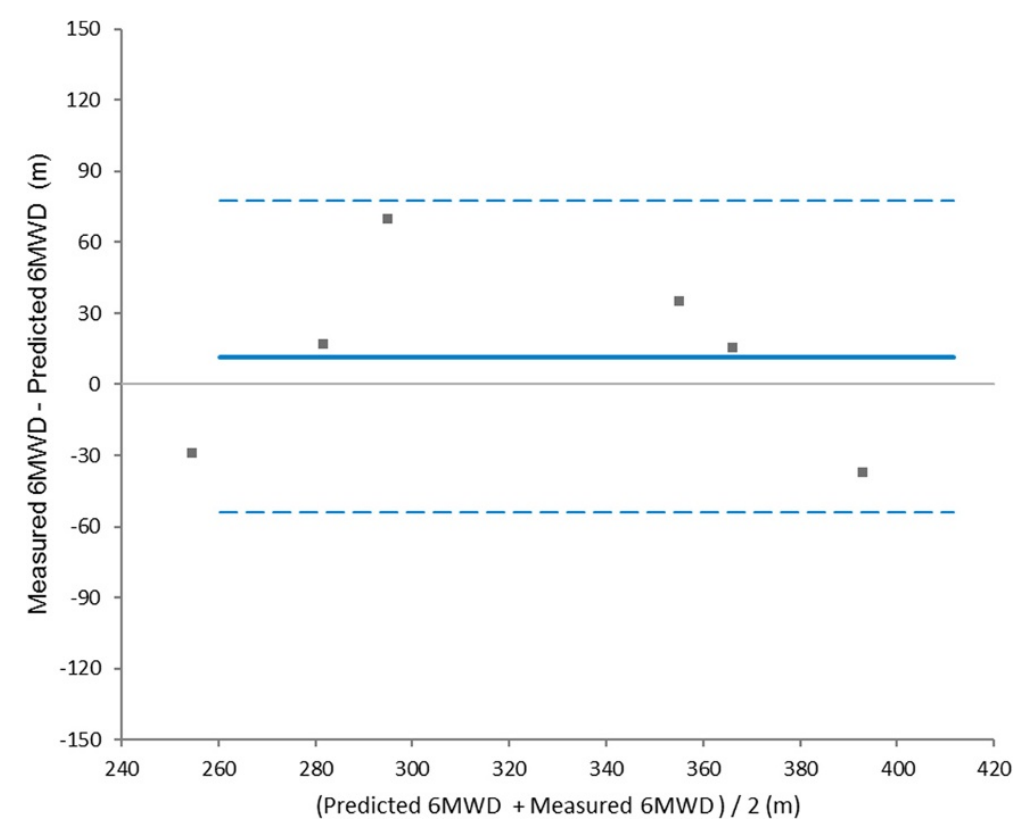

Figure 2 Representation of Bland and Altman Measure of the Walked Distance in the 6-minute Walk Test (6MWD) and the predicted distance by the proposed equation.

variables (FVC and FVE1) were not represented in the final model, probably due to the homogeneity of the sample.

The combination of independent variables was able to explain $86 \%$ of the variability in $6 \mathrm{MWD}$ in the total sample, which is very satisfactory in comparison with previously published equations for healthy individuals. A previous study found models that explained $42 \%$ and $38 \%$ of the variation in $6 \mathrm{MWD}$ for healthy men and women, respectively [21]. Other factors that were not assessed in this study may, however, have influenced the 6MWD: percentage of lean body mass, quadriceps strength, leg length, level of physical activity prior to cardiac surgery $[26,27]$.

\section{Limitation of the study}

Our study has some potential limitations such as 6MWD assessment before surgery and did not include other variables such as the characterization of the physical activity level before surgery that could influence the functional status of patients. Moreover, the interpretation of our data should be cautious to represent patients from a cardiology service alone.

\section{Conclusion}

In this study, the determinants of 6MWD at hospital discharge in patients undergoing cardiac surgery were: the type of surgery, СРB time and the Functional Independence Measure and body mass index. This study generated a reliable equation to predict 6MWD.

\section{Abbreviations}

6MWD: Distance walked in six-minute walk test; 6MWT: Six-minute walk test; BMI: Body mass index; CABG: Coronary artery bypass grafting;

CPB: Cardiopulmonary bypass; FEV1: Forced expiratory volume in one second; FIM: Functional independence measure; FVC: Forced vital capacity;

ICU: Intensive care unit; LVEF: Left ventricular ejection fraction; MV: Mechanical ventilation; NHP: Nottingham health profile.

\section{Competing interests}

The authors declare that they have no competing interests.

\section{Authors' contributions}

GUO, VOC, LPAC, AAAF, MLCN, WMSJ, TCFC and VJSF conceived and planned the activities that led to the study. GUO and LPAC performed assessments. GUO assessed the data. GUO, VOC, MLCN, WMSJ and VJSF prepared the manuscript. All authors read and approved the final manuscript.

\section{Acknowledgements}

The authors would like to thank the collaboration of the staff of the surgical units, intensive care and postoperative hospitalization of Fundação de Beneficência Hospital Cirurgia for this study.

\section{Source of funding}

This study was funded by the National Council for Scientific and Technological Development (CNPq/Brazil) and the Foundation for Research and Technological Innovation Support of the State of Sergipe (FAPITEC-SE).

\section{Author details}

'Master's Degree of the Postgraduation Program in Health Sciences at the Federal University of Sergipe, - UFS, Aracaju, SE, Brazil. ${ }^{2}$ Departamento de Fisioterapia da Universidade Federal de Sergipe - UFS, Aracaju, SE, Brazil. ${ }^{3}$ The GREAT Group (GRupo de Estudos em ATividade física), Aracaju, Brazil. ${ }^{4}$ Curso de Fisioterapia da Universidade Tiradentes - UNIT, Aracaju, SE, Brazil.

${ }^{5}$ Departamento de Fisioterapia da Universidade Federal de Sergipe - UFS, Lagarto, SE, Brazil.

Received: 30 January 2014 Accepted: 6 May 2014

Published: 31 May 2014 


\section{References}

1. Laizo A, Delgado FED, Rocha GM: Complications that increase the time of hospitalization at ICU of patients submitted to cardiac surgery. Rev Bras Cir Cardiovasc 2010, 25(2):166-171.

2. Borges JBC, Ferreira DLM, Carvalho SMR, Martins AS, Andrade RR, Silva MMA: Pain intensity and postoperative functional assessment after heart surgery. Braz J Cardiovasc Surg 2006, 21:393-402.

3. Faggiano P, D'Aloia A, Gualeni A, Brentana L, Dei CL: The 6 minute walking test in chronic heart failure: indications, interpretation and limitations from a review of the literature. Eur J Heart Fail 2004, 6:687-691.

4. Woo MA, Moser DK, Stevenson LW, Stevenson WG: Six-minute walk test and heart rate variability: lack of association in advanced stages of heart failure. Am J Crit Care 1997, 6:348-354.

5. Cacciatore F, Abete P, Mazzella F, Furgi G, Nicolino A, Longobardi G, Testa G, Langellotto A, Infante T, Napoli C, Ferrara N, Rengo F: Six-minute walking test but not ejection fraction predicts mortality in elderly patients undergoing cardiac rehabilitation following coronary artery bypass grafting. Eur J Cardiovasc Prev Rehabil 2012, 19:1401-1409.

6. La Rovere MT, Pinna GD, Maestri R, Olmetti F, Paganini V, Riccardi G, Riccardi R, Goggi C, Ranucci M, Febo O: The 6-minute walking test and all-cause mortality in patients undergoing a post-cardiac surgery rehabilitation program. Eur J Prev Cardiol 2013, [Epub ahead of print].

7. De Feo S, Tramarin R, Faggiano P, Ambrosetti M, Riccio C, Diaco T, Carlon R, Temporelli PL, Baroni PL, Fattirolli F, Griffo R: The inabilitytoperform a 6 minute walkingtestaftercardio-thoracicsurgeryis a markerofclinicalseverityandpooroutcome. Data fromthe ISYDE 2008 Italiansurvey. Int J Cardiol 2011, 151:115-116.

8. ATS Committee on Proficiency Standards for Clinical Pulmonary Function Laboratories: ATS statement: guidelines for the six-minute walk test. Am J Respir Crit Care Med 2002, 166(1):111-117.

9. Miller MR, Hankinson J, Brusasco V, Burgos F, Casaburi R, Coates A, Crapo R, Enright P, van der Grinten CP, Gustafsson P, Jensen R, Johnson DC, Maclntyre N, McKay R, Navajas D, Pedersen OF, Pellegrino R, Viegi G, Wanger J, ATS/ERS Task Force: ATS/ERS Task Force. Standardisation of spirometry. Eur Respir J 2005, 26:319-338.

10. Ottenbacher KJ, Hsu Y, Granger CV, Fiedler RC: The reliability of the functional independence measure: a quantitative review. Arch Phys Med Rehabil 1996, 77(12):1226-1232.

11. Riberto M, Miyasaki HM, Jucá SHH, Sakamoto H, Pinto PPN, Battistella LR: Validação da versão brasileira da Medida de Independência Funcional. Acta Fisiátrica 2004, 11:72-76.

12. Montagnani $G$, Vagheggini $G$, Panait Vlad E, Berrighi $D$, Pantani $L$, Ambrosino N: Use of the functional independence measure in people for whom mechanical ventilation is difficult. Phys Ther 2011, 91(7):1109-1115.

13. Teixeira-Salmela LF, Magalhães Lde C, Souza AC, Lima Mde C, Lima RC, Goulart F: Adaptation of the Nottingham Health Profile: a simple measure to assess quality of life. Cad Saude Publica 2004, 20:905-914.

14. Haan R, Aaronson N, Limburg M, Langton Hewer R, van Crevel H: Measuring quality of life in stroke. Stroke 1993, 24:320-327.

15. Bland JM, Altman DG: Statistical methods for assessing agrément between two methods of clinical measurement. Lancet 1986, 1:307-310.

16. Fiorina C, Vizzardi E, Lorusso R, Maggio M, De Cicco G, Nodari S, Faggiano P, Dei Cas $L$ : The 6-min walking test early after cardiac surgery. Reference values and the effects of rehabilitation programme. Eur J Cardiothorac Surg 2007, 32:724-729.

17. Enright PL, McBurnie MA, Bittner V, Tracy RP, McNamara R, Arnold A, Newman AB: The 6-min walk test: a quick measure of functional status in elderly adults. Chest 2003, 14:387-398.

18. Enright PL, Sherrill DL: Reference equations for the six-minute walk in healthy adults. Am J Respir Crit Care Med 1998, 158:1384-1387.

19. Cimen S, Ozkul V, Ketenci B, Yurtseven N, Gunay R, Ketenci B, Gercekoglu H, Demirtas M: Daily comparisonofrespiratoryfunctionsbetweenonpumpandoffpumppatientsundergoing CABG. Eur J Cardiothorac Surg 2003, 23:589-594.

20. Cox CM, Ascione R, Cohen AM, Davies IM, Ryder IG, Angelini GD: Effectofcardiopulmonarybypassonpulmonarygasexchange: a prospectiverandomizedstudy. Ann Thorac Surg 2000, 69:140-145.

21. Iwama AM, Andrade GN, Shima P, Tanni SE, Godoy I, Dourado VZ: The six-minute walk test and body weight-walk distance product in healthy Brazilian subjects. Braz J Med Biol Res 2009, 42(11):1080-1085.

22. Ben Saad H, Prefaut C, Tabka Z, Mtir AH, Chemit M, Hassaoune R, Ben Abid T, Zara K, Mercier G, Zbidi A, Hayot M: 6-minute walk distance in healthy
North Africans older than 40 years: influence of parity. Respir Med 2009, 103(1):74-84.

23. Camarri B, Eastwood PR, Cecins NM, Thompson PJ, Jenkins S: Six minute walk distance in healthy subjects aged 55-75 years. Respir Med 2006, 100:658-665.

24. Mak VH, Bugler JR, Roberts CM, Spiro SG: Effect of arterial oxygen desaturation on six minute walk distance, perceived effort, and perceived breathlessness in patients with airflow limitation. Thorax 1993, 48(1):33-38

25. Wijkstra PJ, TenVergert EM, van der Mark TW, Postma DS, Van Altena R, Kraan J, Koëter GH: Relation of lung function, maximal inspiratory pressure, dyspnea, and quality of life with exercise capacity in patients with chronic obstructive pulmonary disease. Thorax 1994, 49(5):468-472.

26. Troosters T, Gosselink R, Decramer M: Six minute walking distance in healthy elderly subjects. Eur Respir J 1999, 14:270-274.

27. Poh H, Eastwood PR, Cecins NM, Ho KT, Jenkins SC: Six-minute walk distance in healthy Singaporean adults cannot be predicted using reference equations derived from Caucasian populations. Respirology 2006, 11:211-216.

doi:10.1186/1749-8090-9-95

Cite this article as: Oliveira et al: Determinants of distance walked during the six-minute walk test in patients undergoing cardiac surgery at hospital discharge. Journal of Cardiothoracic Surgery 2014 9:95.

\section{Submit your next manuscript to BioMed Central and take full advantage of:}

- Convenient online submission

- Thorough peer review

- No space constraints or color figure charges

- Immediate publication on acceptance

- Inclusion in PubMed, CAS, Scopus and Google Scholar

- Research which is freely available for redistribution

Submit your manuscript at www.biomedcentral.com/submit
C Biomed Central 